\title{
EVALUACIÓN DE LAS HERRAMIENTAS TECNOLÓGICAS PARA EL APRENDIZAJE DEL INGLÉS EN LA UTE Y LA PERCEPCIÓN DE LOS ESTUDIANTES
}

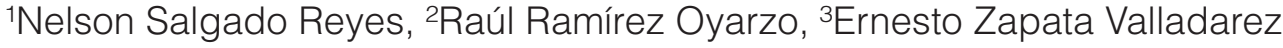 \\ 1Universidad Tecnológica Equinoccial, Quito - Ecuador, nelson.salgado@ute.edu.ec \\ 2Universidad Tecnológica Equinoccial, Quito - Ecuador, rramirez@ute.edu.ec \\ 3Universidad Tecnológica Equinoccial, Quito - Ecuador, Izapata@ute.edu.ec
}

Recepción / Received: 14,10,2015

Aceptación / Accepted: 15,12,2015

Publicado / Published: 30,12,2015

\begin{abstract}
Resumen:
La investigación tiene como fin presentar una evaluación de las herramientas de aprendizaje del inglés en la Universidad Tecnológica Equinoccial y la percepción que tienen los estudiantes en relación al uso de estas herramientas, así como de las TICs. La investigación parte del análisis de la importancia que radican las herramientas tecnológicas en el aprendizaje de los idiomas. Se verifica que el uso de las tecnologías de la información incluida en el uso de software especializado, favorecen el aprendizaje debido a la interacción con el audio y video, además de hacer uso de herramientas acorde a la práctica actual. El estudio continua con el análisis de las plataformas virtuales UTE en línea y la herramienta Cambridge LMS, donde se determina sus ventajas y desventajas además de analizar comparativamente los beneficios y carencias de cada uno. La investigación se profundiza con una encuesta que incluyó a un total de 3072 estudiantes, que permitió conocer la percepción de los estudiantes sobre el uso de las herramientas y en general acerca de la metodología y el aprendizaje del inglés en la UTE. El estudio concluye por una parte en la importancia que tiene el uso de las herramientas tecnológicas en el aprendizaje del inglés en la UTE. Existe también la necesidad de fortalecer el uso de las plataformas, complementadas con actividades prácticas que pueden ser incluidas en la plataforma UTE, lo cual favorecería al aprendizaje del inglés en los estudiantes incrementando la calidad educativa en la Universidad Tecnológica Equinoccial.
\end{abstract}

Palabras clave: TICs, herramientas de aprendizaje, inglés, UTE en línea, Cambridge LMS.

\begin{abstract}
:
The research aims to provide an assessment of the tools of learning English in the Universidad Tecnológica Equinoccial (UTE) and the students' perception regarding the use of these tools, as well as ICTs. The research part of the analysis of the importance of technological tools lie in learning languages. It verified that the use of information technologies including the use of specialized software, promote learning due to the interaction with the audio and video, besides making use of tools according to current practice.. The study continues with the analysis of virtual platforms and online UTE Cambridge LMS tool, where its advantages and disadvantages determined in addition to comparatively analyze the benefits and shortcomings of each. The research is deepened by a survey that included a total of 3072 students, which allowed to know the perception of the students on the use of tools and in general about the methodology and learning English in the joint venture. The study concludes by a party to the importance of the use of technology in learning English in the UTE tools. There is also the need to strengthen the use of platforms, complemented with practical activities that can be included in the joint venture platform, which favor the learning of English in increasing the quality of education students at the UTE.
\end{abstract}

Keywords: ICT, learning tools, English, UTE online, Cambridge LMS. 


\section{INTRODUCCIÓN}

Las TICs "Tecnologías de la Información y de la Comunicación", se presentan hoy en día en todas las sociedades, provocando una transformación en la educación, principalmente de uso práctico en el aprendizaje de lenguas extranjeras. Entre sus principales ventajas está la motivación del estudiante, fomentar el proceso individual de aprendizaje, la retroalimentación inmediata y el acceso no lineal a la información (Salgado, 2005). La informática ha logrado reproducir en un alto grado las condiciones de uso natural de la lengua mediante el empleo de una serie de herramientas como son: el correo electrónico, las salas de charla (chats), los foros, las listas de distribución (mailinglists), los videos, plataformas, diapositivas, internet; herramientas que apoyan el modelo lingüístico de adquisición de segundas lenguas entendida como acción y comunicación (Ministerio de Educación de España, 2010). De acuerdo a Contreras (2012:iv), "las características de la realidad virtual que recrea Internet hacen de ésta una herramienta casi 'natural' para el proceso de enseñanza-aprendizaje, puesto que la capacidad de simulación facilita el aprendizaje".

Las TICs se han convertido en una herramienta utilizada constantemente para el aprendizaje de un idioma (Sparks, 2006). El uso adecuado de las TICs permite que el aprendizaje sea complementario y activo. Sin embargo, la integración de las TICs en el proceso enseñanza-aprendizaje es un proceso complejo y multidimensional que incluye muchas dinámicas como herramientas TICs, profesor, estudiantes, administración escolar, programas educativos y cultura de la institución (Demiraslan \& Usluel, 2006). Cuando las TICs entra en el entorno socio-cultural de la entidad, puede desencadenar cambios en las actividades, currículo y en las relaciones interpersonales en el ambiente de aprendizaje y esta recíprocamente afectado por los cambios que el mismos causas (Demiraslan \& Usluel, 2008).

Es por ello que el presente estudio tiene como objeto realizar un análisis de las herramientas de aprendizaje utilizadas por la UTE, lo que permitirá por una parte entender las ventajas y desventajas en este caso particular, del uso de las plataformas UTE en línea y la herramienta Cambridge, para el aprendizaje del inglés en la universidad, y por otra parte conocer la percepción de estudiantes acerca de importancia del uso de las TICs, así como su aplicación.

Siendo que el alcance del presente artículo se limita al análisis de la modalidad presencial.

\section{EVALUACIÓNDELASHERRAMIENTAS TECNOLÓGICAS EN LA UTE}

El modelo pedagógico en la UTE tiene como objetivo la formación integral del estudiante, a través de su activa participación dentro los procesos de enseñanza y aprendizaje de lenguas y culturas extranjeras.

El modelo busca potencializar conocimientos, actitudes, capacidades, valores y competencias, que le permitan solucionar problemas propios de su área profesional, al igual que de su vida cotidiana, en un idioma extranjero, haciendo énfasis en la interacción comunicativa y el desarrollo de competencias lingüísticas apoyados en la incorporación de las TICs que facilita aprendizaje significativo.

El curso de idiomas está dividido en 8 niveles basados en el Marco Común Europeo que empieza desde un nivel básico A1 hasta el B2.

El proceso de enseñanza-aprendizaje se alinea con las habilidades que presenta el Marco Común Europeo, como son: Listening, Reading, Speaking and Writing (escuchar, leer, hablar y escribir). Al finalizar los ocho niveles el estudiante será capaz de describir actividades que hace por sí mismo o alguien más las hace por él o ella. Puede describir experiencias y eventos, metas, objetivos, y situaciones hipotéticas, tanto en presente como en pasado y futuro, sustentado con razones, significativas, coherentes, así como explicaciones y opiniones claras y lógicas.

Los componentes del programa de inglés presencial contienen: el Folleto Gramatical, Folleto de Lectura, Folleto de Comprensión auditiva.

El programa electrónico de aprendizaje, es un programa de apoyo que trabaja con información online seleccionada, para los niveles B2 y C1 y que aporta al aprendizaje de inglés con un fuerte enfoque en hablar y escuchar. Se puede usar el curso dentro y fuera del aula con una mezcla de 
tecnología. El programa electrónico es utilizado tanto a nivel presencial como no presencial.

Dentro del programa electrónico, las plataformas virtuales que se utilizan son UTE en línea y Cambridge.

\section{ANÁLISIS DE PLATAFORMAS UTE EN LÍNEA Y CAMBRIDGE LMS}

UTE en línea.- La Universidad Tecnológica Equinoccial dispone de una plataforma en línea a través de la cual es posible mantener una comunicación más directa del estudiante con la universidad. El LMS es una plataforma virtual, cuyo objetivo es presentar los planes de estudio de cada materia, pone a disposición de los estudiantes lecturas de interés de acuerdo a cada subtema de estudio. Permite además una comunicación más dinámica entre profesores y estudiantes, ya que brinda servicios de mensajería, foros, chat, planificación de actividades a lo largo del curso, recepción de tareas, etc. que facilitan el proceso de enseñanza y aprendizaje.

Entre otros, los servicios que presta la plataforma al estudiante son: consulta de notas, horarios, documentos, impedimentos, datos económicos, evaluación de profesores, información de la universidad, matrículas, clases virtuales, encuestas, videos. Permite acceder a los diferentes cursos y recursos de las asignaturas. En la figura 1 se presenta parte del interfaz que permite el acceso a las principales herramientas de uso estudiantes, de la plataforma



Figura 1. Interfaz visual herramientas UTE en línea

\section{Análisis.-}

Se puede decir de la plataforma UTE de manera general, que la misma, es una herramienta muy útil que permite ofrecer a los estudiantes acceso a todos los programas, cursos, herramientas de la universidad y a través de la cual se puede hacer la entrega de evaluaciones y además tener acercamiento con los docentes y en ciertos casos con estudiantes.

La accesibilidad es relativamente sencilla, pero tiene gran cantidad de ítems que van haciendo más complejo su uso, aunque la universidad lo utiliza para que los estudiantes tengan acceso a los diferentes recursos de la universidad en línea.

Específicamente para la asignatura de inglés le permite al estudiante el ingreso de trabajos, deberes, rendición de exámenes y una pocas herramientas adicionales como foros y comunicación con el maestro, sin embargo no otorga herramientas de soporte significativas adicionales para el aprendizaje del inglés a través de esta plataforma, sino más bien se utiliza como herramienta necesaria para la evaluación.

\section{Cambridge.-}

La plataforma Cambridge cubre las necesidades pedagógicas y curriculares de la Universidad y facilita trabajar en el mundo virtual y electrónico de la enseñanza de inglés, cubriendo los niveles desde el nivel B1 hasta el C1.

La metodología Touchstone Online Course está estructurada por cada unidad de cuatro lecciones con actividades en cada unidad, en cada unidad el estudiantes debe desarrollar sus habilidades tanto en la gramática, vocabulario, audición, lectura y la escritura.

Esta herramienta permite asesorar a cada estudiante permitiendo que se establezca retroalimentación de forma instantánea ya que la metodología es constructivista siendo un aprendizaje basado en 'learning outcomes' o aprendizaje de competencias, objetivos directamente relacionados con el CEF (Common European Framework o Marco Común Europeo). Además permite la planificación, implementación y evaluación del curso, abarcando los estándares internacionalmente establecidos del CEF.

Además el programa permite:

- Planificación, implementación y evaluación del curso, abarcando los estándares internacionalmente establecidos del Marco Común Europeo (CEF), 
- Whiteboard Software o aplicación para pizarra interactiva - para todos los niveles. Funciona muy bien en una pizarra interactivay también con un proyector solamente.

- Testcrafter Software a aplicación para la creación de pruebas parciales y exámenes finales, (Banco de 2000 preguntas).

- Los materiales de apoyo para los profesores, tales como la instalación del software para la pizarra interactiva, audio CDs, videos, manuales de profesor (del libro en papel y la plataforma), etc. con la debida renovación

Como ejemplo se presentan algunas de las fases de aprendizaje de la plataforma (Ver figuras 2, 3 y 4).

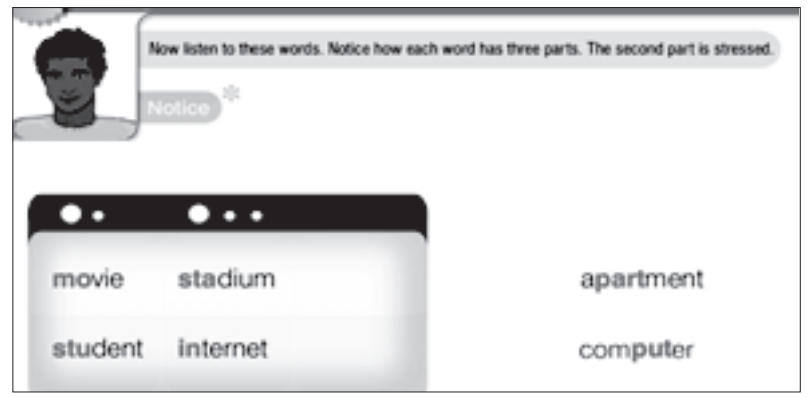

Figura 2. Reglas de pronunciación

Se aprende las reglas de la pronunciación para poder entender mejor cuando escuchen y para poder hablar con claridad. Se aprende las reglas de la pronunciación para poder entender mejor cuando escuchen y para poder hablar con claridad.

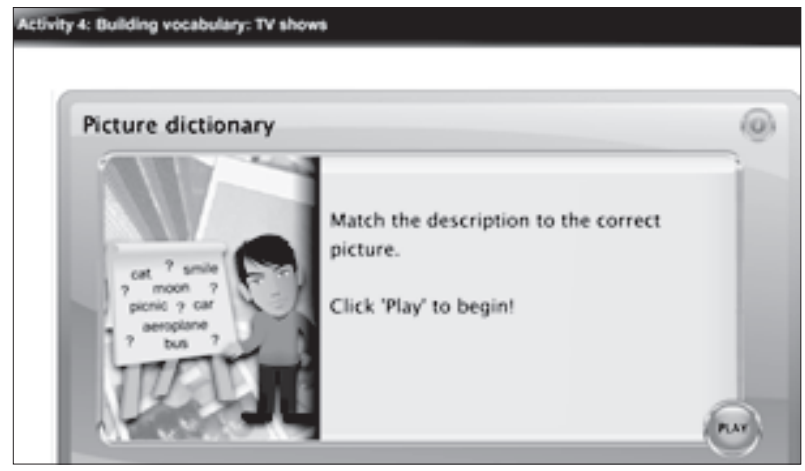

Figura 3. Vocabulario

Se aprende el vocabulario nuevo con videos, actividades y juegos, los cuales les hacen usar el vocabulario en contexto significativo, reciclar y repasar.

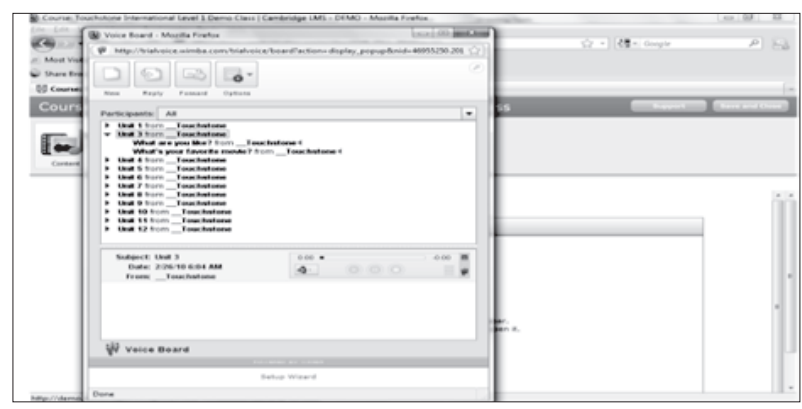

Figura 4. Publicación de voz

Se puede grabar la voz y publicar las grabaciones para poder escuchar los otros estudiantes, entre varias otras herramientas.

\section{ANÁLISIS COMPARATIVO}

La herramienta UTE en línea es muy versátil, permite integrar módulos, actividades, programa de aprendizaje, lo cual le otorga gran ventaja, sin embargo en la actualidad no dispone de un programa diseñado específicamente para el aprendizaje del inglés como lo tiene Cambridge que es diseñado específicamente para este propósito.

Por tanto es claro que ambos programas deben trabajar de manera integrada para complementar el aprendizaje del inglés, como actualmente se lo está haciendo, aunque con algunos recursos es posible mejorar la metodología en general.

Cambridge es un software completo para el aprendizaje y que ha sido diseñado con una estructura para cubrir con los niveles de aprendizaje exigidos a nivel internacional. Posee varias falencias que se pudo detectar en su análisis, para el tipo de aprendizaje universitario como son las evaluaciones, además aunque dispone de prácticas de speaking y chat para interrelación, esto no reemplaza en su totalidad las conversaciones e interacción personal, pero en general es un software completo para el aprendizaje, por lo que adelantándose a los resultados de la investigación, se puede indicar que éste programa debe ser la base del aprendizaje tanto a nivel presencial, como a distancia, mientras que la herramienta UTE en línea, aunque ha sido solamente un medio de comunicación entre la universidad y los estudiantes, pero que a su vez le permite acceder a foros, comunicación, envío de trabajos y sobre todo que tiene la posibilidad de incluir prácticas por su flexibilidad y por el mismo hecho de haber sido diseñado para ello, puede ser el espacio para insertar actividades que fortalezcan el uso de la 
herramienta de aprendizaje, integrando prácticas, foros y actividades.

Comparación de herramientas.- A continuación se presenta a través de una matriz una comparación entre las herramientas Cambridge y UTE en línea (Tabla 1), ambas actualmente utilizadas complementariamente para el aprendizaje del inglés peroqueasuvezpueden sercomparadasensus características como herramientas técnicas, aplicación, usabilidad y los aspectos pedagógicos para el aprendizaje del inglés en laUTE.

Tabla 1. Análisis comparativo UTE en línea - Cambridge

\begin{tabular}{|c|c|c|c|}
\hline & Cambridge & $\begin{array}{l}\text { UTE en } \\
\text { línea }\end{array}$ & Observaciones \\
\hline \multicolumn{4}{|c|}{ HERRAMIENTAS TÉCNICAS } \\
\hline Diseño & Establecido & $\begin{array}{l}\text { Modificable a } \\
\text { gusto }\end{array}$ & \\
\hline Modificación & $\begin{array}{l}\text { No } \\
\text { modificable }\end{array}$ & Modificable & \\
\hline $\begin{array}{ll}\text { Recursos } & y \\
\text { videos } & \end{array}$ & $\begin{array}{l}\text { Dispone de la } \\
\text { información } \\
\text { necesaria } \\
\text { para los } \\
\text { módulos }\end{array}$ & $\begin{array}{l}\text { No dispone } \\
\text { de } \\
\text { información } \\
\text { por módulos }\end{array}$ & $\begin{array}{l}\text { Cambridge es } \\
\text { diseñado para el } \\
\text { aprendizaje del } \\
\text { inglés, una } \\
\text { aplicación en } \\
\text { UTE en línea de } \\
\text { videos, audio } \\
\text { sería difícil y } \\
\text { costoso de } \\
\text { integrar. }\end{array}$ \\
\hline $\begin{array}{l}\text { Integración de } \\
\text { cronogramas, } \\
\text { seguimiento, } \\
\text { notas }\end{array}$ & No integra & $\begin{array}{l}\text { Tiene } \\
\text { integrado } \\
\text { actualmente y } \\
\text { puede } \\
\text { incrementarse }\end{array}$ & \\
\hline Accesibilidad & $\begin{array}{ll}\text { Facilidad en } \\
\text { el acceso }\end{array}$ & $\begin{array}{ll}\text { Facilidad en } \\
\text { el acceso }\end{array}$ & \\
\hline \multicolumn{4}{|c|}{ APLICACIÓN EN LA UTE } \\
\hline $\begin{array}{l}\text { Uso del } \\
\text { programa por } \\
\text { el estudiante }\end{array}$ & \begin{tabular}{l}
\multicolumn{2}{l}{ Permanente } \\
en el \\
programa de \\
inglés
\end{tabular} & $\begin{array}{l}\text { Utilizado } \\
\text { cuando es } \\
\text { requerido }\end{array}$ & \\
\hline $\begin{array}{l}\text { Actividades } \\
\text { prácticas }\end{array}$ & $\begin{array}{l}\text { Establecidas } \\
\text { por el } \\
\text { programa, } \\
\text { dispone de } \\
\text { varias } \\
\text { actividades } \\
\text { de acuerdo al } \\
\text { nivel }\end{array}$ & $\begin{array}{l}\text { Establecidas } \\
\text { por el maestro } \\
\text { en base a su } \\
\text { criterio o se } \\
\text { puede } \\
\text { integrar de } \\
\text { acuerdo a la } \\
\text { necesidad }\end{array}$ & $\begin{array}{l}\text { UTE en línea } \\
\text { permite integrar } \\
\text { las actividades } \\
\text { prácticas de } \\
\text { acuerdo a la } \\
\text { necesidad, pero } \\
\text { con la } \\
\text { complejidad de } \\
\text { que han que } \\
\text { crearlas. } \\
\text { Cambridge ya } \\
\text { dispone de las } \\
\text { aplicaciones, } \\
\text { pero de acuerdo } \\
\text { a su programa. }\end{array}$ \\
\hline \multicolumn{4}{|c|}{ CARACTERÍSTICAS DE USABILIDAD } \\
\hline $\begin{array}{ll}\text { Soporte } & y \\
\text { ayuda } & \end{array}$ & No dispone & $\begin{array}{l}\text { Dispone de } \\
\text { ayuda y } \\
\text { comunicación } \\
\text { con maestros } \\
\text { y otros } \\
\text { recursos }\end{array}$ & \\
\hline Comunicación & $\begin{array}{l}\text { No permite } \\
\text { comunicación } \\
\text { interestudiant } \\
\text { es, con } \\
\text { maestros a la } \\
\text { universidad }\end{array}$ & $\begin{array}{l}\text { Si permite la } \\
\text { comunicación } \\
\text { interestudiant } \\
\text { es, con } \\
\text { maestros a la } \\
\text { universidad }\end{array}$ & \\
\hline $\begin{array}{l}\text { Función } \\
\text { independiente } \\
\text { para } \\
\text { enseñanza del } \\
\text { inglés }\end{array}$ & $\begin{array}{l}\text { Puede ser una } \\
\text { herramienta } \\
\text { independiente } \\
\text { y única con la } \\
\text { cual se puede }\end{array}$ & $\begin{array}{l}\text { No funciona } \\
\text { independiente } \\
\text {, no dispone } \\
\text { de módulos } \\
\text { de }\end{array}$ & \begin{tabular}{ll}
\multicolumn{2}{l}{ Cambridge } \\
puede ser la \\
herramienta \\
única & de \\
aprendizaje & de
\end{tabular} \\
\hline
\end{tabular}

Evaluación crítica de las plataformas actualmente utilizadas (UTE en línea y Cambridge).- La metodología planteada se centra en las actuales plataformas que utiliza la universidad, sin embargo es importante analizar de manera crítica a ambas plataformas, con el fin de que nuevos estudios consideren la posibilidad de mejorar la plataforma de la universidad, así como detectar las potenciales falencias de la plataforma Cambridge para suplir las mismas con las demás herramientas.

Plataforma de aprendizaje de inglés Cambridge.No es fácil considerar mejoras en la plataforma de aprendizaje Cambridge, pues la misma no depende de la universidad, sino que depende directamente del proveedor, además en general esta plataforma es bastante completa y de nivel internacional, sin embargo es importante considerar de manera crítica los aspectos que tiene falencias de acuerdo a la observación realizada y la percepción que tienen los estudiantes y maestros.

\section{Falencias detectadas en la plataforma Cambridge:}

- Muchas de las preguntas de práctica que presenta la plataforma solamente ponen opciones múltiples que en muchos casos pueden ser contestadas por descarte, es decir existen dos opciones de respuesta en cada caso y en muchos casos la segunda opción no tiene sentido. Además si el estudiante no contestó correctamente, en la segunda oportunidad simplemente podrá contestar directamente por la segunda opción, con lo cual no es posible realmente evaluar si el estudiante está aprendiendo o no.

- Se puede intuir que un estudiante sin mucho interés por el aprendizaje podría solamente aprender a manejar el programa sin realmente aprender $e$ ir pasando los niveles, claro esto implicaría problemas en la evaluación, pero estas evaluaciones al ser una calificación porcentual, no necesariamente son determinantes en el paso de nivel de los estudiantes.

- $\quad$ La plataforma al igual que la mayor parte de las metodologías del inglés hacen mayor énfasis en el inglés escrito y de este se evalúa, mientras que la comunicación en inglés, principalmente considerada como la producción oral no se evalúa a profundidad.

De este análisis en algún momento dado se podrá sugerir al proveedor o complementar el estudio y evaluaciones con aspectos metodológicos que complementen las falencias observadas. 
Plataforma UTE.- Al igual que en el análisis previo, la plataforma UTE está definida y es la herramienta a través de la cual se trabaja con un relativo éxito en relación a comunicación general con los estudiantes, sin embargo el análisis de la plataforma mediante su observación sumado a las percepciones de estudiantes y maestros muestra que no se integran herramientas para el aprendizaje del inglés, solamente se utiliza para envío de trabajos, pero los mismos usualmente son mayormente teóricos y no prácticos.

Dentro de esta plataforma se puede incluir videos, foros, chats, datos de contacto para iniciar conversaciones con estudiantes de otras universidades, entre otros recursos.

\section{INVESTIGACIÓN DE CAMPO}

Para complemento del estudio, se desarrolló una encuesta, misma que se aplicó a través de la herramienta UTE en línea a un total de 3072 estudiantes, obteniéndose por tanto un censo estudiantil, con resultados confiables acerca de la metodología y el aprendizaje del inglés en la UTE.

Entre los principales resultados de la encuesta se presentan los siguientes a continuación:

Pregunta: ¿De acuerdo a su punto de vista, de qué manera influyen los recursos tecnológicos utilizados en un programa de estudio del inglés?

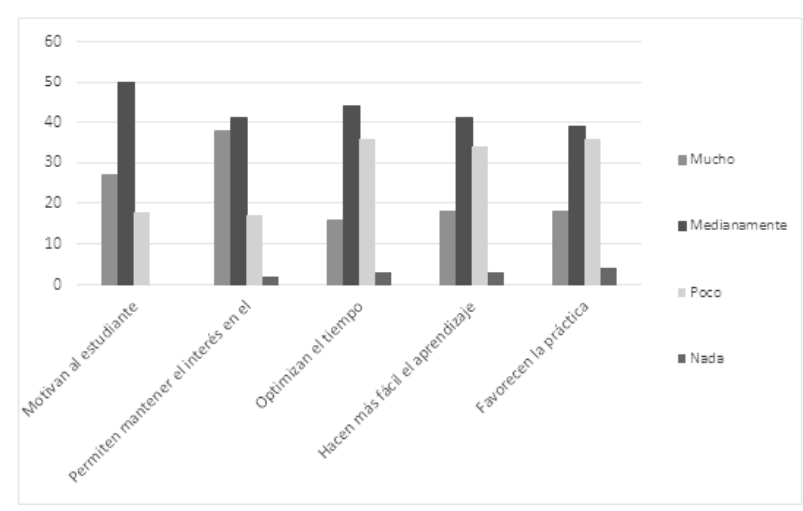

Figura 5. Influencia de los recursos tecnológicos

En la figura 5 se observa que las principales opiniones indican que las herramientas tecnológicas permiten mantener el interés y motivan al estudiante, aunque existen variedad de opiniones.
Al consultar: ¿De qué manera se utilizan las siguientes herramientas tecnológicas en el programa de educación del inglés en la UTE?, los resultados fueron:

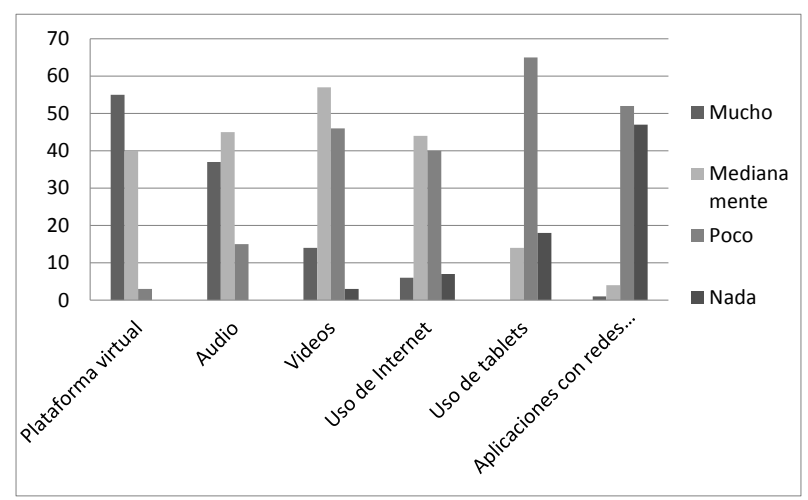

Figura 6. Utilización de herramientas

Es claro que un alto porcentaje indica que mayormente utilizan las herramientas como la plataforma virtual y el audio como principales herramientas (ver figura 6), mientras que indican que medianamente utilizan videos, internet, pero muy poco o nada tablets o aplicaciones con redes sociales.

Al consultar: De los siguientes aspectos califique de acuerdo a su criterio cómo favorecen al aprendizaje del inglés los siguientes factores:

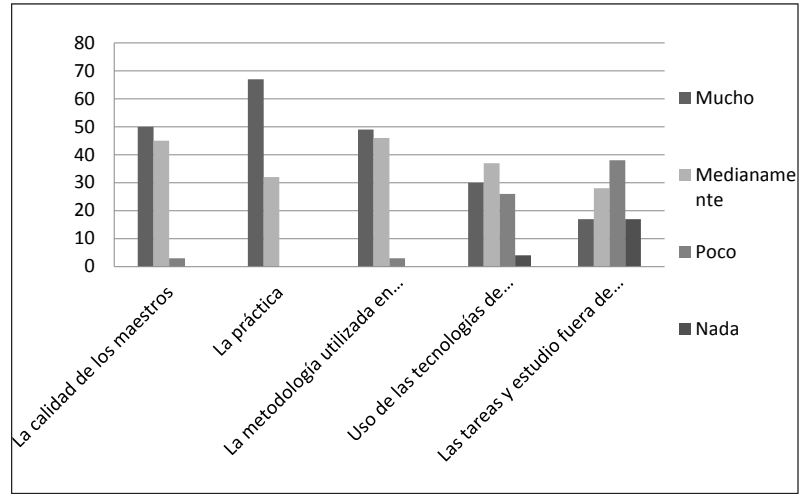

Figura 7. Aprendizaje de ingles

La mayor parte de estudiantes consideran principalmente 3 aspectos que son: la calidad de los maestros, la práctica y la metodología de enseñanza en clase (ver figura 7). En menor proporción aunque aún con una proporción alta indican que las tecnologías de información pueden afectar medianamente o mucho en el aprendizaje del inglés. Pocos consideran las tareas y estudio fuera de clase.

Finalmente a la pregunta: ¿De acuerdo a su punto de vista, considera que el uso de la plataformas tecnológica Cambridge utilizada en la UTE es: 


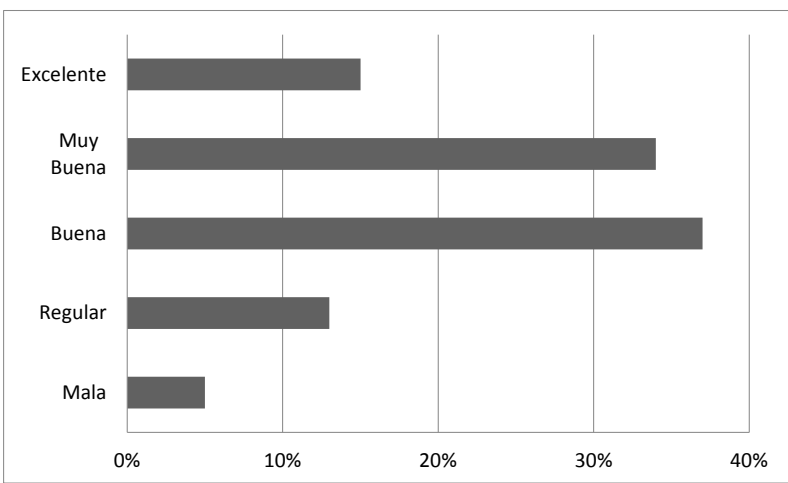

(Figura 8. Uso de la plataforma Cambridge)

Como se observa en la figura 8, para la mayor parte de los estudiantes el uso de la plataforma tecnológica Cambridge es buena o muy buena, más no excelente.

Los resultados de la investigación primaria muestran que a pesar de que los estudiantes están conscientes de las bondades de las TICs en el aprendizaje del inglés, no están completamente satisfechos con el aprendizaje, esto se debe principalmente a que no se utiliza en su máximo potencial las plataformas, contrariamente se hace mayor uso de recursos como las clases magistrales, lo cual limita los alcances o ventajas del uso de las plataformas, por lo que finalmente los estudiantes están de acuerdo en su totalidad el programa que se utiliza en la UTE.

Al profundizar en las respuestas de los estudiantes, se pudo observar que existen varias sugerencias que indican los estudiantes acerca de hacer más prácticas, haciendo uso de videos, películas, conversación con extranjeros, entre varios otros aspectos que fortalecerían su aprendizaje

\section{CONCLUSIONESY RECOMENDACIONES}

Es muy importante y se puede considerar como una ventaja importante el uso de un LMS estructurado en la Universidad Tecnológica Equinoccial, más aún cuando el mismo está basado en la evaluación del Marco Común Europeo.

La plataforma UTE mostró ser una herramienta muy útil y bastante versátil que además de integrar y favorecer los procesos de comunicación, le permite a la universidad la creación de nuevos complementos para el mejoramiento de los aprendizajes, específicamente en el inglés, sin embargo actualmente la herramienta no se ha potencializado para la asignatura de inglés.
Al analizar en los estudiantes el resultado de la aplicación de las herramientas tecnológicas, así como aprendizaje del inglés, se ha mostrado que a pesar de que los estudiantes están conscientes de la importancia del uso de las TICs, las mismas no se están utilizando en todo su alcance, por lo que no existe una satisfacción completa de los estudiantes en el aprendizaje. Pero es claro también que con ciertos ajustes en la metodología, así como complementando la práctica del inglés, se podría mejorar los resultados en el aprendizaje de los estudiantes.

El uso de la plataforma Cambridge por parte de los estudiantes es limitado y parcial, dando mayor énfasis a las clases magistrales, en la educación presencial del inglés, por lo que no se potencia en su totalidad el uso de la plataforma virtual Cambridge y por lo que se sugiere el uso en su totalidad.

Es importante incorporar herramientas adicionales que fortalezcan la práctica en el inglés, que den soporte al actual trabajo del docente en el aula, las cuales pueden ser integradas a la plataforma UTE en línea como las siguientes: Prácticas específicas de vocabulario con escritura y pronunciación. Así mismo que se envíe trabajos que incluyan: videos, comunicación entre estudiantes por audio (skype), foros, películas en inglés, debates, traducción de texto en inglés, redacciones de investigación en inglés.

De esta manera, si bien el presente estudio ha cumplido con ser una evaluación de las plataformas educativas utilizadas en la UTE, se presenta las alternativas de mejoramiento, a través de herramientas que se pueden incorporar haciendo uso de herramientas tecnológicas abiertas existentes y que pueden accederse a través del computador fácilmente, con lo cual será posible mejorar el aprendizaje y beneficiar a la universidad y a cada uno de los estudiantes.

\section{AGRADECIMIENTOS}

Se hace reconocimiento especial a la Universidad Tecnológica Equinoccial por su apertura a la investigación y por la entrega de información para realizar un análisis crítico en favor del mejoramiento de la calidad educativa. 


\section{REFERENCIAS}

Demiraslan, Y. \& Usluel, Y. (2008). Proceso de integración de las TICs. Australasian Journal of Educational Technology. Kızlırmak: Ani Publishing. 24 (4), 458-474.

Ministerio de Educación de España. (2010). E proceso de integración y uso pedagógico de las TIC en los centros educativos. Un estudio de casos. Revista de educación: Las TICs en la Educación obligatoria: de la teoría a la práctica. (352). 77-97.

Sparks, C. (2006). La influencia de Internet en los medios de comunicación convencionales. La ventana global. Madrid: Taurus.

Contreras, N. (2012). La enseñanza-aprendizaje de lenguas extranjeras y las TICs: el caso del Español como Lengua Extranjera (ELE). España: Universidad de Jaen, revista electrónica. Recuperado de: http:// revistaselectronicas.ujaen.es/index.php/ ininv/article/view/233/214

Salgado, E. (2005). Estrategias de enseñanza virtual universitaria. San José: Editorial ULACIT 